Paula Huth, ${ }^{1 *}$ Samantha E. Wirth, ${ }^{2}$ Deborah Baker, ${ }^{2}$ David C. Nicholas, ${ }^{3}$ Aphrodite Douris, ${ }^{4}$ Jennifer Freiman, ${ }^{5}$ Kelly E. Kline, ${ }^{6}$ Katelynn Devinney, ${ }^{7}$ Sabine Gläsker ${ }^{8}$ and Colin Schwensohn ${ }^{9}$

'New York State Dept. of Health, Bureau of Communicable Disease Control, Empire State Plaza, Corning Tower, Floor 6, Albany, NY 12237, USA

${ }^{2}$ New York State Dept. of Health, Wadsworth Center, David Axelrod Institute,120 New Scotland Ave., Albany, NY 12208, USA

${ }^{3}$ New York State Dept. of Health, Bureau of Community Environmental Health and Food Protection, Empire State Plaza, Corning Tower, Floor 13, Albany, NY 12237, USA ${ }^{4}$ U.S. Dept. of Agriculture, Food Safety and Inspection Service, 950 College Station Road, Athens, GA 30605, USA 5U.S. Dept. of Agriculture, Food Safety and Inspection Service, 355 E St. SW, Washington, D.C. 20024, USA EPennsylvania Dept. of Health, Bureau of Epidemiology, Room 933, Health and Welfare Bldg., Harrisburg, PA 17120, USA

${ }^{7}$ New York City Dept. of Health and Mental Hygiene, Bureau of Communicable Disease, Queens, NY 11101, USA ${ }^{8}$ New York City Dept. of Health and Mental Hygiene, Public Health Laboratory, New York, NY 10016, USA

IU.S. Centers for Disease Control and Prevention, Division of Foodborne, Waterborne and Environmental Diseases, 1600 Clifton Road, MS-A38, Atlanta, GA 30333, USA
PEER-REVIEWED ARTICLE

Food Protection Trends, Vol. 41, No. 2, p. 239-245 Copyright 2021 , international Association for Food Protection 2900100 th Street, Suite 309, Des Moines, IA 50322-3855

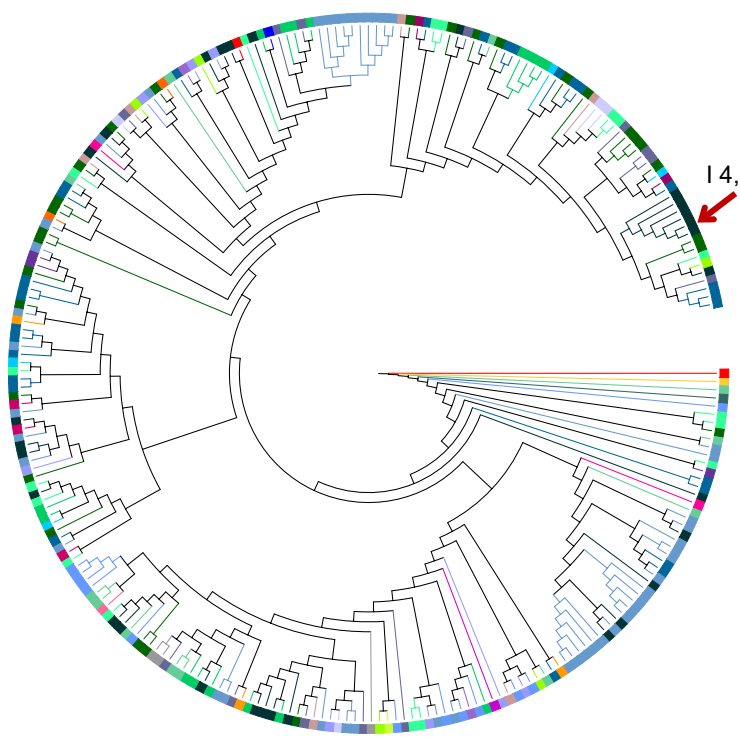

\title{
Ability of Whole-Genome Sequencing to Refine a Salmonella I 4,[5],12:i:- Cluster in New York State and Detect a Multistate Outbreak Linked to Raw Poultry
}

\section{ABSTRACT}

Whole-genome sequencing (WGS) has proven to be a more powerful tool than pulsed-field gel electrophoresis for foodborne illness cluster definition because of improved resolution. Between November 2017 and May 2018, the New York State (NYS) Dept. of Health investigated 10 cases of Salmonella I 4,[5],12:i:- with pulsed-field gel electrophoresis pattern JPXX01.0621; comparison of case exposures did not identify a common source of infection. In June 2018, the NYS Dept. of Health's Wadsworth Center analyzed the isolates using WGS and defined a subcluster of five isolates related within zero to six single-nucleotide polymorphisms. The National Center for Biotechnology Information Pathogen Detection browser advanced this investigation by identifying additional clinical and food (chicken) isolates related within zero to eight single-nucleotide polymorphisms to the original subcluster. Comparison of WGS-related isolates would support the hypothesis that illness was associated with exposure to a kosher poultry product. This outbreak ultimately consisted of 25 cases from six states. Of 20 cases interviewed, all reported chicken consumption, and of those able to recall brand information, $83 \%$ cited a brand produced at a facility linked to the WGS-related chicken isolates. This paper demonstrates how WGS was able to refine a Salmonella I 4,[5],12:i:- cluster in NYS to uncover a multistate outbreak linked to raw poultry.

\section{INTRODUCTION}

In the United States, foodborne illness remains a key public health concern. The Centers for Disease Control and Prevention (CDC) estimate that annually in the United States, one in six individuals experiences foodborne-related illness. The burden of foodborne illness is considerable; approximately 48 million illnesses, 128,000 hospitalizations, and 3,000 deaths result from the consumption of contaminated food nationally each year (2). In particular, nontyphoidal Salmonella spp. are a common cause of foodborne-related illness in the United States, and worldwide, causing an estimated 1.2 million illnesses, 23,000 hospitalizations, and 450 deaths in the United States annually. The source for about 1 million of these illnesses is through the consumption 
of contaminated food, predominantly of animal origin (4). The timely identification of disease clusters and attribution of foodborne illness cases to specific food sources is vital to halting disease transmission, preventing additional cases of human illness, and developing prevention and control strategies to improve food safety. To that end, and in an effort to improve surveillance for enteric disease outbreaks, laboratory subtyping methods to determine the relatedness of clinical isolates have become a rapidly evolving science, with various advanced molecular techniques on the horizon.

Epidemiologists and laboratorians at state and local health departments nationwide, including those at the New York State Department of Health (NYSDOH), transitioned in 2019 from using pulsed-field gel electrophoresis (PFGE) to whole-genome sequencing (WGS) for enteric disease surveillance and outbreak detection. Although PFGE can show relatedness among isolates in a cluster, WGS is able to provide added resolution for assessing genetic relatedness. As such, WGS analysis is based on the inference that closely related isolates likely share a common ancestor and the corresponding cases likely had exposure to a common source of infection. The NYSDOH Wadsworth Center Laboratory (WCL) began using PFGE for enteric disease cluster detection on a national scale in 1997 and implemented WGS for local cluster surveillance in 2013. In March 2019, NYSDOH WCL discontinued the use of PFGE for isolate subtyping. This transition from PFGE-WGS tandem analysis to analysis exclusively by WGS has required constant communication among NYSDOH epidemiologists, public health laboratorians, and environmental health specialists to determine workflow and thresholds for enteric disease outbreak investigations of local and national scale.

By way of the Food Safety Modernization Act (2011), NYSDOH, in partnership with Cornell University, was established as an Integrated Food Safety Center of Excellence (NY CoE) in 2015 with a mission to support, enhance, and improve foodborne outbreak investigations at state and metropolitan public health departments across the nation. NY CoE, a recognized subject matter expert in WGS, has made it a priority to advance knowledge on the subject, particularly among those in public health who interpret WGS results for cluster detection and investigation. Training and education efforts to improve food safety and assist with cluster identification are multidisciplinary in approach, targeting epidemiologists, public health laboratorians, and environmental health specialists. This investigation (i) describes how WGS was able to define a subcluster of cases within an endemic Salmonella serotype I 4, [5],12:i:- cluster in New York state (NYS) linked to a commercially available raw poultry product; (ii) highlights how WGS can narrow the focus of an epidemiology investigation, particularly when highly related clinical and food isolates have been identified; (iii) demonstrates successful communication of WGS analysis and collaboration among local, state, and federal partners; and (iv) demonstrates how WGS, along with epidemiologic information, provided a strong foundation for communication with consumers and industry.

\section{MATERIALS AND METHODS}

\section{Salmonella surveillance: laboratory}

Clinical laboratories in NYS, exclusive of New York City (NYC), are required to submit confirmed Salmonella isolates to the NYSDOH WCL for confirmatory testing, subtyping, and serotyping. At the time of this investigation, PFGE analysis was still being performed at the NYSDOH WCL. Results of the PFGE analysis were uploaded through BioNumerics (Applied Maths, Austin, TX) to the PulseNet National Database for comparison with national PFGE data. WGS and bioinformatic data for all NYS Salmonella serotype Typhimurium isolates (including I 4,[5],12:i:-) were analyzed via an in-house-developed, reference-based single-nucleotide polymorphism (SNP) pipeline for local cluster detection (8). Salmonella Typhimurium LT2 was used as a reference genome to map the sequence reads and find positions with SNPs. For a SNP to be identified, a genome position was required to have at least $20 \times$ depth of coverage of high-quality mapped reads with $95 \%$ of the reads in agreement. SNPs that failed these requirements or that mapped over phage-associated islands and repeat were excluded. In addition, sequence data were shared with CDC PulseNet through the web-based utility BaseSpace (Illumina, Inc.) to facilitate PulseNet enteric disease surveillance activities. The sequence data, along with minimal sample metadata, were also uploaded to the National Center for Biotechnology Information (NCBI) and analyzed on the NCBI Pathogen Detection isolates browser, which enabled searching for genetically related clinical, food, and environmental isolates on a national level (13). The sequence data and metadata for isolates included in the study can be accessed on NCBI using the accession numbers provided in Table 1.

\section{Salmonella surveillance: epidemiology}

Reporting of all culture-confirmed cases of salmonellosis in NYS is mandated under the NYS Sanitary Code (10NYCRR 2.10 and 2.14) and the NYC Health Code. All cases are reported electronically to the NYSDOH, and it is the responsibility of the local health department of the case's county of residence to initiate follow-up. All cases of salmonellosis are contacted by a local health department official and interviewed using a detailed questionnaire to capture demographic data, clinical illness and medical care details, and a 7-day (before illness onset) history of food, water, animal, and travel exposures. Since 2016, NYSDOH has offered interviewing assistance to local health departments with completion of Salmonella initial hypothesis generation and/or outbreak questionnaires. 


\begin{tabular}{|c|c|c|c|c|}
\hline Strain ID & BioSample Accession $^{0}$ & SRA Run Accession ${ }^{+}$ & Isolation Source & Collection Date \\
\hline PNUSAS027931 & SAMN08134123 & SRR6371606 & stool & $10 / 17 / 17$ \\
\hline PNUSAS026252 & SAMN08134060 & SRR6380788 & stool & $9 / 26 / 2017$ \\
\hline NY84671838 & SAMN08513397 & SRR6761221 & ground turkey & 2018 \\
\hline NY84671873 & SAMN08513396 & SRR6761464 & ground turkey & 2018 \\
\hline PNUSAS037576 & SAMN09429145 & SRR7346617 & stool & $3 / 6 / 2018$ \\
\hline PNUSAS036486 & SAMN08964172 & SRR7049885 & stool & $2 / 22 / 2018$ \\
\hline PNUSAS034333 & SAMN08964080 & SRR7049814 & stool & $1 / 23 / 2018$ \\
\hline PNUSAS038526 & SAMN09430482 & SRR7348240 & stool & $2 / 28 / 2018$ \\
\hline PNUSAS030104 & SAMN08513667 & SRR6703791 & stool & $11 / 15 / 2017$ \\
\hline PNUSAS040819* & SAMN09260941 & SRR7218800 & stool & $4 / 2 / 2018$ \\
\hline \multicolumn{5}{|c|}{$\begin{array}{l}{ }^{0} \text { unique identifier to access sample metadata. } \\
{ }^{+} \text {unique identifier to access sample sequence data within the SRA (sequence read archive). } \\
{ }^{*} \text { related within 4-7 SNPs from isolates in WGS Cluster } 1 \text { (not pictured in Fig. 1). }\end{array}$} \\
\hline
\end{tabular}

Once requested, either a NYSDOH epidemiologist or a trained student interviewer team member will attempt case contact. For initial interviews, a minimum of three call attempts are completed during business hours, followed by at least one attempt afterhours (evening or weekend) before closeout as lost to follow-up. For outbreak or focused questionnaire interviews, in addition to the multiple daytime attempts, at least two afterhours attempts are completed before closeout. State and student team assistance has had a tremendous positive impact on interview completion rates for salmonellosis cases in NYS overall and played a key role in data collection for the investigation described here. A case was defined as an individual with an infection of Salmonella I 4,[5],12:i:- pattern JPXX01.0621, highly related to the WGS outbreak clade within 0 to 8 SNPs, and with an isolate collection date on or after 1 September 2017.

\section{RESULTS}

In November 2017, NYSDOH began investigating two cases of Salmonella I 4,[5],12:i:- with isolates matching PFGE pattern JPXX01.0621. By May 2018, NYSDOH WCL had identified 10 cases of Salmonella I 4,[5],12:i:- in NYS (excluding NYC) with PFGE pattern JPXX01.0621. A retrospective look at surveillance data indicated that this number of cases, within the given timeframe, was not above the baseline average for this PFGE pattern in the state. Each case was contacted by the NYSDOH via phone, and all cases consented to interview using an extensive hypothesisgenerating questionnaire consisting of questions regarding grocery shopping locations, foods eaten outside the home, food allergies, and special diets. For the 7-day food history before illness onset, cases were questioned about 120 food items (poultry, seafood, eggs, dairy, cheese, beef, other meat or meat alternatives, vegetables, herbs, fruit, frozen foods, nuts, cereals, and processed foods), in addition to other exposure pertaining to sources of drinking water, travel history, and animal exposures. Cases were geographically dispersed across the state, and available epidemiologic exposure histories did not yield leading hypotheses about the source of infection among cases.

In June 2018, NYSDOH WCL used WGS data generated from an in-house bioinformatic pipeline to further distinguish the genetic relatedness among the 10 case isolates involved in the cluster. The initial report sent by NYSDOH WCL to NYSDOH epidemiology staff illustrates how a single PFGE cluster type can be resolved by WGS (Fig. 1). Within the Salmonella I 4,[5],12:i:- PFGE pattern JPXX01.0621 cluster, WGS analysis defined a subcluster of four clinical isolates, which were highly related within 0 to 6 SNPs (WGS cluster 1) and would later be linked to a common food source. Three other clinical isolates included in the original PFGE cluster (PNUSAS036486 and WGS cluster 3) appeared genetically unrelated to the isolates in WGS cluster 1 , distanced by 12 to 14 and 66 to 72 SNPs, respectively. Isolates in WGS cluster 2 (of ground turkey origin) also appeared genetically unrelated to the isolates in WGS cluster 1 (44 to 62 SNP difference) and furthermore were not isolates previously associated with the original PFGE cluster. One additional clinical isolate associated with the original PFGE cluster (PNUSAS040819, not pictured) was from a NYS 


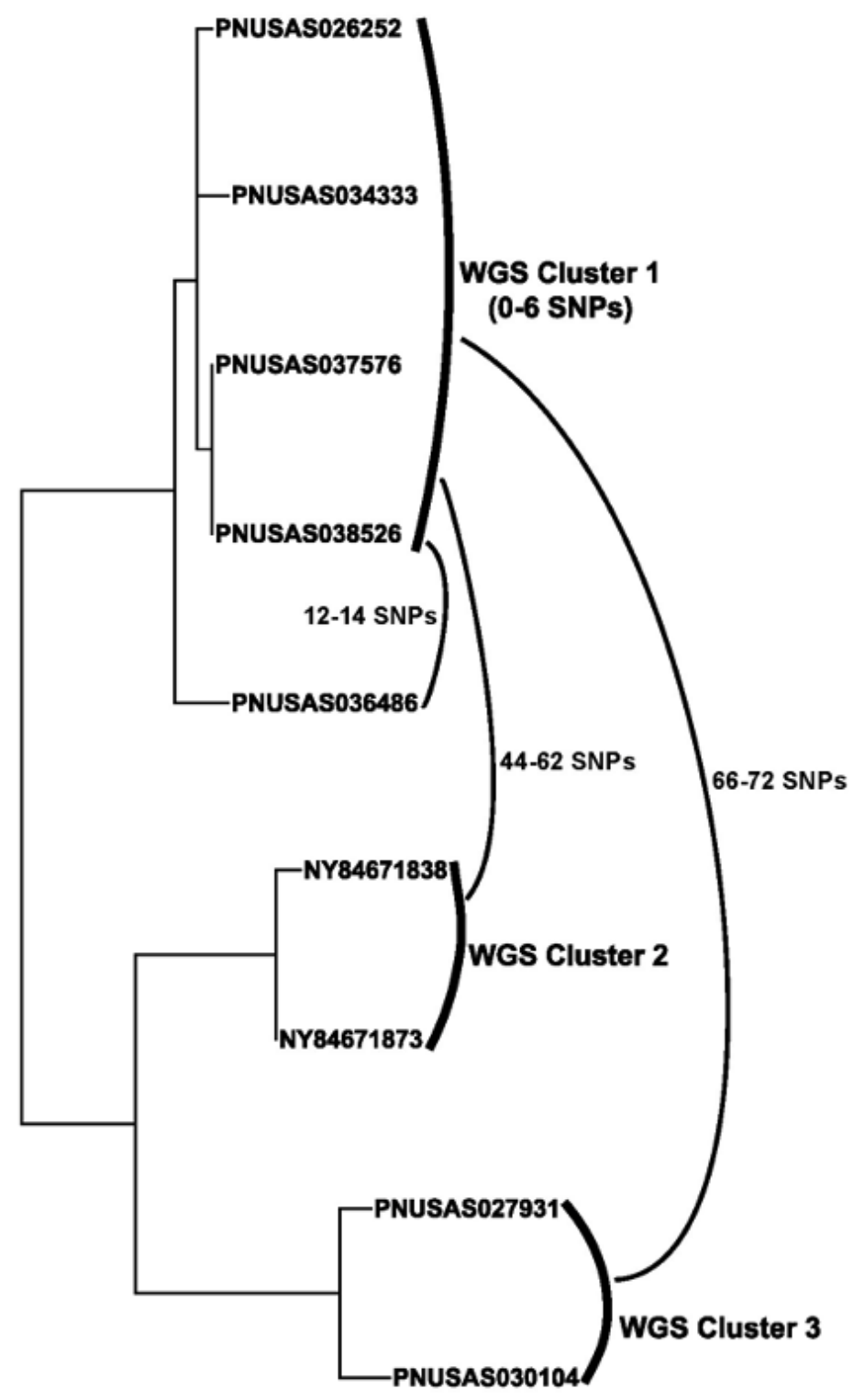

Figure 1. Initial whole-genome sequencing report from the New York State Dept. of Health Wadsworth Center Laboratory, illustrating further resolution of a Salmonella I 4,[5],12:i:- cluster, PFGE pattern JPXX01.0621.

resident who was tested by the NYC Department of Health and Mental Hygiene and was related within 4 to 7 SNPs from isolates in WGS cluster 1 (13). Among the five cases whose isolates comprised WGS cluster 1 (including PNUSAS040819), onset dates ranged from 25 September 2017 to 29 March 2018. The age range was 1 to 76 years, with a median of 61 years. Three of five $(60 \%)$ cases were male. Two cases were hospitalized (40\%), and no deaths were reported. Cases resided in four separate counties across the state. Comparison of the subset of cases included in WGS cluster 1 suggested a potential association with exposure to a kosher food item. One case reported a kosher diet; two cases cited unrelated kosher grocery stores among their list of food shop- ping locations; and two cases reported exposure to unrelated food products with a kosher brand name. A kosher association was not identified among the food isolates included in WGS cluster 2 or among the cases included in WGS cluster 3.

In an attempt to identify additional isolates genetically related to those in WGS cluster 1, epidemiologists at the NYSDOH queried the NCBI Pathogen Detection browser (13). The browser detected six additional clinical isolates and six food isolates highly related by WGS to the isolates comprising WGS cluster 1 (Table 2). All identified isolates (Salmonella I 4,[5],12:i:-) matched PFGE pattern JPXX01.0621. The clinical isolates, collected from cases residing in New York (one), NYC (two), Maryland (one), 


\section{TABLE 2. Additional S. I 4,[5],124i:- isolates identified as highly related to WGS Cluster 1 using the NCBI Pathogen Detection Isolates Browser}

\begin{tabular}{l|c|c|c|c} 
Strain ID & Isolation Source & Location & Collection Date & $\begin{array}{c}\text { SNP Diversity Range } \\
\text { when Compared with } \\
\text { Isolates in WGS Cluster 1 }\end{array}$ \\
\hline PNUSAS037581 & stool & NY & $3 / 5 / 2018$ & $0-6$ \\
\hline PNUSAS041017 & stool & NYC & $3 / 19 / 2018$ & $0-8$ \\
\hline PNUSAS041014 & stool & NYC & $3 / 7 / 2018$ & $0-8$ \\
\hline PNUSAS035615 & stool & PA & $1 / 14 / 2018$ & $0-8$ \\
\hline PNUSAS039728 & stool & VA & $4 / 5 / 2018$ & $0-8$ \\
\hline PNUSAS040730 & stool & MD & $4 / 16 / 2018$ & $0-8$ \\
\hline FSIS11807011 & chicken & NJ & $12 / 21 / 2017$ & $0-6$ \\
\hline FSIS21821073 & chicken & PA & $1 / 29 / 2018$ & $0-6$ \\
\hline FSIS21821453 & chicken & PA & $4 / 3 / 2018$ & $0-6$ \\
\hline FSIS31800041 & chicken & PA & $12 / 27 / 2017$ & $0-6$ \\
\hline FSIS31800132 & chicken & USA:PA & $1 / 29 / 2018$ & $0-6$ \\
\hline FSIS31800355 & chicken & USA:NY & $4 / 10 / 2018$ & $0-6$ \\
\hline
\end{tabular}

Pennsylvania (one), and Virginia (one), were within 0 to 8 SNPs of the isolates belonging to WGS cluster 1 . The food isolates originated from the U.S. Department of Agriculture's (USDA's) Food Safety and Inspection Service (FSIS) sampling of chicken carcass rinse, chicken livers, ground chicken, and chicken breast in New Jersey (one), Pennsylvania (four), and New York (one) and were within 0 to 6 SNPs from the clinical isolates belonging to WGS cluster 1 .

On 15 June 2018, NYSDOH contacted epidemiology colleagues at NYC, Maryland, Pennsylvania, and Virginia departments of health to request exposure information on cases whose isolates were related to WGS cluster 1 . The initial interview data gathered from these cases suggested a kosher food or kosher diet association. Of the 11 cases now included in the multistate WGS cluster, 10 cases had been interviewed with a Salmonella hypothesis-generating questionnaire. All cases (10 of 10) reported chicken consumption. Six cases $(60 \%)$ reported kosher food exposure or exposures. Eight cases $(80 \%)$ reported exposure to dairy, and six cases (60\%) reported exposure to cucumbers, zucchini, or squash. Clinical isolates had collection dates spanning September 2017 to April 2018.

On 18 June 2018, the NYSDOH Bureau of Community Environmental Health and Food Protection contacted FSIS to request additional information about the six chicken isolates related by WGS on NCBI to the clinical isolates belonging to WGS cluster 1 (13). FSIS shared information about the isolates, including product and brand information, in accordance with established information-sharing processes
(11). The isolates, collected in December 2017 through April 2018 , originated from samples of raw chicken collected at multiple slaughter and processing establishments, including samples collected as part of routine testing under the Salmonella performance standards (9).

FSIS notified NYSDOH on 20 June 2018 that all six chicken isolates were products of kosher or halal processing facilities. Recognizing the strength of the combined epidemiologic, environmental health, and laboratory data, NYSDOH immediately contacted CDC to provide a summary of the multistate Salmonella I 4, [5],12:i:investigation and the hypothesis that the source of illness was a kosher-related food product, possibly chicken. In agreement with CDC, a focused questionnaire was developed by NYSDOH and distributed to NYC, Maryland, Pennsylvania, and Virginia for case re-interviews. All cases were contacted via phone and asked to participate in a second interview focusing on special diets and exposure to kosher or halal food items. For the 7-day food history before illness onset, cases were questioned about 17 food items (including chicken, fish, products containing eggs, dairy, fresh produce, prepared salads, deli meats, and spreads and dips). One aim of the focused questionnaire was to probe for more detailed poultry exposures; under the poultry section, 25 brand names were inserted as a checklist to assist cases with recall of brand information. This information would later be used to determine whether there was an association between products produced at facilities where WGS-related chicken isolates were collected and human illness. 
By 13 July 2018, 10 of 11 cases had been re-interviewed. All 10 cases (100\%) reported chicken consumption, and 7 cases $(70 \%)$ reported following a kosher diet. Cases reported yes $(60 \%)$ or maybe $(30 \%)$ to exposure to kosher or halalprocessed chicken. In addition, 6 cases $(60 \%)$ could recall chicken brand information, with 5 cases (50\%) reporting exposure to Brand $\mathrm{X}$ kosher poultry during the week before illness onset. Brand X kosher poultry was produced at one of the kosher processing facilities where FSIS isolates were collected that were closely related to clinical isolates by WGS.

Active laboratory surveillance for cases throughout July and August 2018 identified six additional WGS-related clinical isolates for epidemiologic follow-up. Of the cases able to be contacted for re-interview $(n=14), 100 \%$ reported chicken exposure. In the interview, 11 of 14 cases were questioned about kosher chicken exposure specifically, of which 9 cases (82\%) reported yes to exposure. Of these cases, 7 individuals reported exposure to Brand X kosher poultry $(78 \%)$. Because of the genetic relatedness ( 0 to 8 SNPs) between isolates of clinical cases reporting exposure to Brand X kosher poultry and the FSIS chicken isolates from the facility that produced Brand X, FSIS proactively contacted the company that produced Brand $\mathrm{X}$ in early August 2018 to make it aware of the ongoing investigation (10). In late August, CDC and FSIS publicly reported the link between illnesses in the outbreak and Brand X kosher poultry products and provided consumer messaging reiterating safe handling and cooking practices for raw chicken products $(1,12)$.

At investigation closeout in December 2018, the outbreak consisted of 25 cases from six states (Massachusetts, 2; Maryland, 1; New York, 16; Ohio, 1; Pennsylvania, 4; and Virginia, 1) (3). Estimated illness onset dates ranged from September 2017 to August 2018. The median age of cases was 16 years (range $<1$ to 75 years); $50 \%$ were female. Of 22 cases, 11 (50\%) were hospitalized, and one death was reported. The severity of this illness was higher than expected, as the typical hospitalization rate among people with Salmonella infection is approximately $22 \%$ (6). All interviewed cases $(n=20)$ reported chicken consumption during the week before illness onset. Of those recalling brand information, $83 \%$ (10 of 12 ) of cases reported exposure to Brand X kosher poultry.

\section{DISCUSSION}

This multistate cluster was initially detected by the NYSDOH in June 2018 as a subset of cases within an endemic Salmonella serotype I 4,[5],12:i:- PFGE-based cluster in NYS (pattern JPXX01.0621) highly related by WGS within 0 to 8 SNPs. PFGE analysis was not able to distinguish this subcluster of genetically related cases. Pattern JPXX01.0621 is a common strain of Salmonella I 4,[5],12:i:and is the most common pattern of the serotype reported to PulseNet, the national subtyping network of public health and food regulatory agency laboratories coordinated by CDC. Based on PulseNet data from 2013 to 2018, an average of 233 isolates (range 175 to 341 ) of Salmonella serotype I 4,[5],12:i:- with PFGE pattern JPXX01.0621 were uploaded to the PulseNet database annually, with this pattern accounting for approximately $10 \%$ of all Salmonella I 4,[5],12:i:- isolates (7). Unlike the PFGE-based case cohort, the cases whose isolates were highly related by WGS shared a tendency to consume kosher food products. A subsequent query using the NCBI Pathogen Detection browser identified additional WGS-related clinical isolates, clustered both temporally and spatially within NYS and from neighboring states (13). The epidemiologic information gathered on these cases supported the link between illness and exposure to a kosher food product. Concurrently, USDA chicken isolates related by WGS to the clinical isolates within 0 to 6 SNPs were identified in the NCBI Pathogen Detection browser. State and federal health partners collaborated to evaluate the evidence and determine that illnesses were associated with consumption of Brand X poultry. Epidemiologic investigations by separate state public health departments indicated kosher chicken exposure as a commonality among cases, specifically Brand X.

Because of compelling epidemiologic, environmental, and laboratory findings, the coordinated response by federal health officials was swift. Based on the available epidemiologic and laboratory information linking illnesses to Brand X kosher raw poultry, on 24 August 2018, FSIS issued a public health alert notifying consumers that Brand $\mathrm{X}$ kosher raw poultry in commerce may be contaminated with Salmonella linked to human illnesses (12). Although there was not sufficient information on the type of chicken products to recommend a recall, the alert notified consumers about the potential health risks associated with the product. On 29 August 2018, CDC posted an investigation notice to inform the public about reports of human illness linked to Brand X kosher raw poultry exposure (1). The notice did not advise consumers to avoid eating Brand X kosher poultry but reinforced practices on how to properly handle and cook raw chicken.

Although exposure to raw poultry is not a novel source of salmonellosis in humans, using WGS as a tool to detect the source of this outbreak was novel (5). Because of the endemic nature of the PFGE pattern involved in this cluster linked to a common exposure, PFGE analysis alone was insufficient to identify the epidemiologically linked subcluster of cases or guide public heath action to prevent additional cases. Integral to the success of this investigation was the use of WGS to differentiate the isolates in a cluster by genetic relatedness, in combination with the capability of the NCBI Pathogen Detection browser to identify related clinical and food isolates not identified locally. Epidemiology, laboratory, and environmental health partners at the local, state, and federal levels worked in unison to ultimately detect 
a Salmonella cluster with a common food contamination source. As we navigate the changing landscape of molecular testing for disease surveillance, it is important to document investigations that warrant a swift multidisciplinary response, specifically when highly related isolates in both food and clinical illness are identified.

\section{ACKNOWLED GMENTS}

The authors thank the NYSDOH Wadsworth Center Applied Genomic Technologies Core for sequencing the isolates listed in Table 1 and the Wadsworth Center Bioinformatics Core for high-quality SNP analysis, in addition to William Wolfgang, Lisa Thompson, Michelle Dickinson, and Amy Saylors from the Wadsworth Center Laboratories.

\section{REFERENCES}

1. Centers for Disease Control and Prevention. 2018. CDC investigation notice regarding a multistate outbreak of Salmonella infections linked to kosher chicken. Available at: https://www.cdc.gov/media/releases/2018/ s0829-salmonella-kosher-chicken.html. Accessed 1 September 2019

2. Centers for Disease Control and Prevention. 2018. Estimates of foodborne illness in the United States. Available at: https://www.cdc. gov/foodborneburden/index.html. Accessed 1 August 2019.

3. Centers for Disease Control and Prevention. 2018. Outbreak of Salmonella infections linked to chicken (final update). Available at: https://www.cdc.gov/salmonella/chicken-08-18/index.html. Accessed 1 September 2019.

4. Centers for Disease Control and Prevention. 2019. Salmonella. Available at: https://www.cdc.gov/salmonella/. Accessed 1 August 2019.

5. Crowe, S. J., A. Green, K. Hernandez, V. Peralta, L. Bottichio, S. Defibaugh-Chavez, A. Douris, L. Gieraltowski, K. Hise, K. La-Pham, K. P. Neil, M. Simmons, G. Tillman, B. Tolar, D. Wagner, J. Wasilenko, K. Holt, E. Trees, and M. E. Wise. 2017. Utility of combining whole genome sequencing with traditional investigational methods to solve foodborne outbreaks of Salmonella infections associated with chicken: a new tool for tackling this challenging food vehicle. J. Food Prot. 80:654-660. https://doi.org/10.4315/0362028X.JFP-16-364.
6. Kennedy, M., R. Villar, D. J. Vugia, T. Rabatsky-Ehr, M. M. Farley, M. Pass, K. Smith, P. Smith, P. R. Cieslak, B. Imhoff, and P. M. Griffin. 2004. Hospitalizations and deaths due to Salmonella infections, FoodNet, 1996-1999. Clin. Infect. Dis. 38:S142-S148. https://doi.org/10.1086/381580.

7. Moreno Switt A. I., Y. Soyer, L. D. Warnick, and M. Wiedmann. 2009. Emergence, distribution, and molecular and phenotypic characteristics of Salmonella enterica serotype 4,5,12:i:-. Foodborne Pathog. Dis 6(4):407-415. https://doi.org/10.1089/ fpd.2008.0213.

8. Taylor, A. J., V. Lappi, W. J. Wolfgang, P. Lapierre, M. J. Palumbo, C. Medus, and D. Boxrud. 2015. Characterization of foodborne outbreaks of Salmonella enterica serovar Enteritidis with whole-genome sequencing single nucleotide polymorphism-based analysis for surveillance and outbreak detection. J. Clin. Microbiol. 53: 3334-33-40. https:// doi.org/10.1128/JCM.01280-15.

9. U.S. Department of Agriculture, Food Safety and Inspection Service. 2016. New performance standards for Salmonella and Campylobacter in not-ready-to-eat comminuted chicken and turkey products and raw chicken parts and changes to related agency verification procedures: response to comments and announcement of implementation schedule. Fed. Reg. 81:7285-7300. Available at: https:// www.federalregister.gov/d/2016-02586. Accessed 27 October 2020.
10. U.S. Department of Agriculture, Food Safety and Inspection Service. 2017. FSIS directive 8080.3 Rev. 2. Foodborne illness investigations. Available at: https:// www.fsis.usda.gov/wps/ wcm/connect/1bffb 125-cd80-4b3a-ab45-1f4c0ad863d9/8080.3.pdf?MOD=AJPERES. Accessed 27 October 2020.

11. U.S. Department of Agriculture, Food Safety and Inspection Service. 2018. FSIS directive 2620.5. Sharing information with state or local agencies, foreign government officials, and international organizations. Available at: https://www.fsis.usda.gov/wps/wcm/ connect/9865dcdf-068c-4e2f-891fef93b879e831/2620.5pdf?MOD=AJPERES Accessed 27 October 2020.

12. U.S. Department of Agriculture, Food Safety and Inspection Service. 2018. FSIS issues public health alert for Empire Kosher Poultry, Inc. raw chicken products due to possible Salmonella contamination. Available at: https://www.fsis.usda.gov/wps/portal/ fsis/ newsroom/newsreleases-statements-transcripts/news-release-archives-by-year/archive/2018/pha-08242018-1. Accessed 27 October 2020.

13. U.S. National Library of Medicine, National Center for Biotechnology Information. 2016. The NCBI pathogen detection project. Available at: https://www.ncbi.nlm.nih.gov/ pathogens/. Accessed 15 August 2019. 